\title{
Prediction of EVAL membrane morphologies using the phase diagram of water-DMSO-EVAL at different temperatures
}

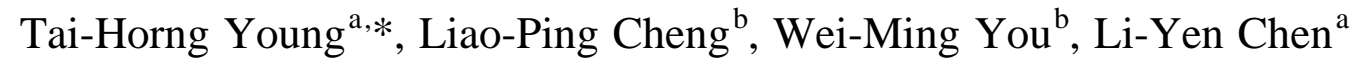 \\ ${ }^{a}$ Graduate Institute of Biomedical Engineering, College of Medicine and College of Engineering, National Taiwan University, Taipei, Taiwan, ROC \\ ${ }^{\mathrm{b}}$ Department of Chemical Engineering, Tamkang University, Taipei, Taiwan, ROC
}

Received 27 April 1998; accepted 2 June 1998

\begin{abstract}
The effects of precipitation temperature on the structures of the EVAL membranes formed in a wet phase inversion process were studied. As the temperature was changed, the phase behavior (liquid-liquid demixing and crystallization boundaries) of the membrane formation system changed accordingly. Therefore, a wide variety of morphologies of the EVAL membranes could be synthesized at different temperatures. At low temperatures (e.g., $25^{\circ} \mathrm{C}$ ), the membrane solution precipitated into a particulate morphology that was governed by the polymer crystallization mechanism, whereas at elevated temperatures (e.g., $65^{\circ} \mathrm{C}$ ) liquid-liquid demixing process dominated the precipitation process and the membrane became cellular in its morphology. In the intermediate cases, the membrane exhibited a structure containing features from both types of phase separations. For the current system, it was found that there existed a good correlation between the phase behavior and the morphology of the membranes. (c) 1999 Elsevier Science Ltd. All rights reserved.
\end{abstract}

Keywords: EVAL membranes; Temperature; Crystallization

\section{Introduction}

The phase inversion process has nowadays become a standard technique to prepare polymeric porous membranes [1]. There is a wealth of literature that describes the mechanism of pore formation in this process, and the central theme of which falls upon the phase demixing process (nucleation and growth) of an initially homogeneous dope solution that is brought into a unstable state by diffusional mass transfer $[1,2]$. For membranes fabricated from precipitation of crystallizable polymers in nonsolvent-solvent systems, the pores are formed by liquid-liquid demixing and/or solidliquid demixing phase separation processes [3-11]. Liquidliquid demixing results in various cellular morphologies whose pores, either sponge- or finger-like, are formed from growth of the polymer-poor phase within the polymer-rich phase. The polymer-rich phase eventually solidifies and becomes the membrane matrix. Solid-liquid demixing originated from crystallization of regular segments of polymer brings particulate features to the morphology of the membrane. Such particulate membranes are

\footnotetext{
* Corresponding author. Tel.: +886-2-23970800 ext. 1455; Fax: +886-2 23940049; E-mail: thyoung@ha.mc.ntu.edu.tw
}

being investigated for possible use in plasma protein separation and microfiltration in our laboratory $[12,13]$. Under ordinary precipitation conditions, crystallization is slower than liquid-liquid demixing because of the time required for orientation of the polymer molecules.

Predictions of the membrane structures dictated by either type of phase separation process are made possible by considerations of the thermodynamic (phase behavior) and kinetic (mass transfer) aspects of the immersion-precipitation process $[5,6,14,15]$. The equilibrium thermodynamics of the ternary system, water-dimethyl sulfoxide (DMSO)poly(ethylene-co-vinyl alcohol) (EVAL), at different temperatures has been described previously [9]. As shown in Fig. 1, there exist two regions of metastability: one with respect to liquid-liquid demixing and the other with respect to crystallization. At $25^{\circ} \mathrm{C}$, the liquid-liquid demixing boundary (i.e., binodal) is located inside the crystallization line. However, because the binodals are less sensitive to temperature changes, these two types of phase transition boundaries are closer at higher temperatures and eventually intersect each other ca. $65^{\circ} \mathrm{C}$. This suggests that membranes prepared at high temperatures tend to exhibit structures derived from liquid-liquid phase separation rather than crystallization. Such a trend is well demonstrated in the 


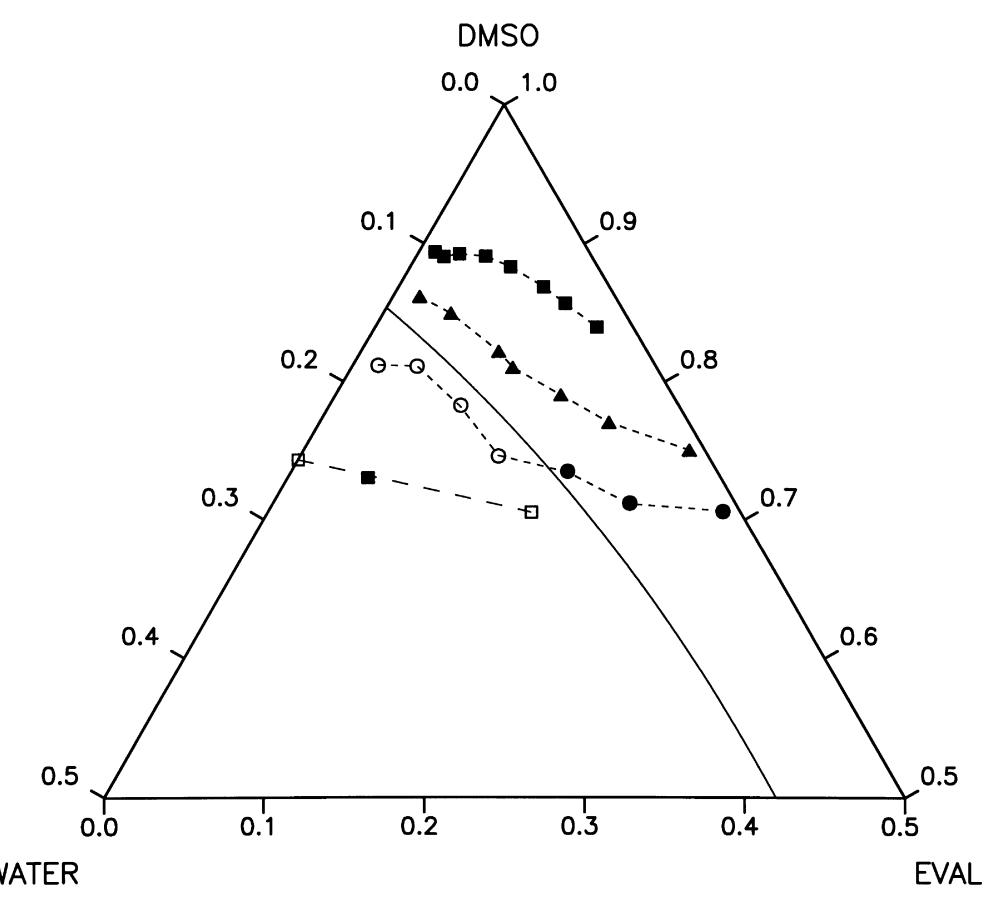

Fig. 1. Phase diagram of water-DMSO-EVAL at various temperatures. - - - : measured crystallization data at $25^{\circ} \mathrm{C}$. $-\mathbf{\Delta}-$ : measured crystallization data

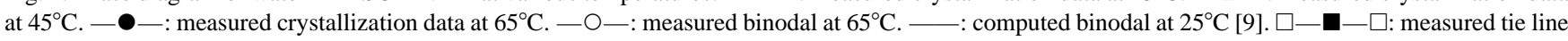
at $65^{\circ} \mathrm{C}$.

membranes precipitated at different temperatures by scanning electron microscopy (SEM). In this work, an attempt has been made to correlate membrane morphology with the phase behavior of the membrane formation system. Equilibrium compositions of the binodal at the precipitation temperature were measured. These data, when combined with the crystallization equilibrium isotherm, illustrated clearly the sequence of precipitation events in the membrane formation process and thereby a good correlation between phase diagram and membrane morphology was discovered for this system. To prevent the occurrence of a finger-macrovoid structure in an instantaneous process, precipitation was carried out in a bath containing a high concentration of solvent. Consequently, for the semicrystalline EVAL a wide variety of morphologies could be obtained ranging from cellular to particulate structures at different temperatures.

\section{Experimental}

\subsection{Material}

The membrane material studied in this work is EVAL which contains ca. 56 mole \% vinyl alcohol. This polymer was kindly supplied by Kuraray Co. Ltd., Japan. Reagent-grade DMSO (Nacalai Tesque, Kyoto, Japan) was used as the solvent for EVAL and was used as received. The nonsolvent for EVAL was deionized and double-distilled water.

\subsection{Equilibrium compositions of the binodal}

A solution with composition falling in the binodal region was prepared at an elevated temperature, and then placed in a constant temperature bath at $65^{\circ} \mathrm{C}$ to induce liquid-liquid demixing. The two equilibrium phases were carefully separated and their weights were measured. The composition of the dilute phase, which contained essentially water and DMSO, was determined by gas chromatography (China Chromatography, 8700T). Knowing the overall composition, the tie line compositions were calculated using mass balance equations.

\subsection{Membrane preparation and characterization}

Membranes were prepared using the direct immersionprecipitation method. An appropriate amount of EVAL was dissolved in DMSO to form a $20 \mathrm{wt} \%$ homogeneous solution. This solution was dispersed uniformly on a glass plate (ca. $200 \mu \mathrm{m}$ ), and then coagulated in a precipitation bath containing $75 \mathrm{wt} \%$ DMSO and $25 \mathrm{wt} \%$ water to form a laminate. The bath with such a high solvent content was a soft coagulant for EVAL, i.e. the membrane formation will be very slow. In order to investigate the effect of temperature on the membrane structure, precipitation was carried out at three different temperatures, 25,45 and $65^{\circ} \mathrm{C}$. The glass plate and the precipitation medium were kept at the same temperature as the polymer solution. The residual nonsolvent and solvent in the nascent membrane were removed by a series of washing steps. The surface and 


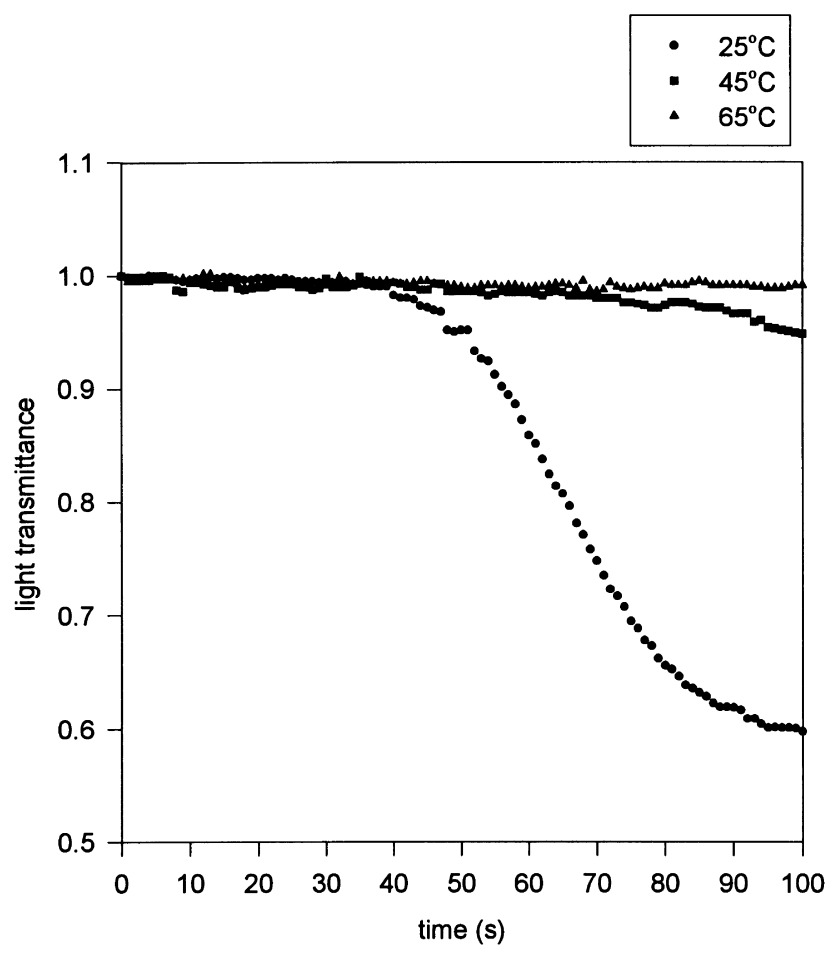

Fig. 2. Light transmission for precipitation of a $20 \mathrm{wt} \%$ EVAL solution in a precipitation bath containing $75 \mathrm{wt} \%$ DMSO and $25 \mathrm{wt} \%$ water at 25,45 and $65^{\circ} \mathrm{C}$.

interior structures of the membrane were examined by SEM (S800, Hitachi).

\subsection{Light transmission measurements}

Light transmission experiments were performed to measure the time for the onset of phase separation in the casting solution at different temperatures. The principle of light transmission experiments is that the light transmittance of the casting solution will decrease with the appearance of optical inhomogenities, which can be induced by liquid-liquid demixing or solid-liquid demixing. A collimated light beam was directed to the membrane solution immersed in a nonsolvent bath. The light transmittance was recorded with a data acquisition system. The light intensity profile was plotted as a function of time. The detailed experimental setup and procedures were shown in a previous publication [6].

\section{Results}

\subsection{Light transmission experiments}

The results of light transmission experiments for precipitation of a $20 \mathrm{wt} \%$ EVAL solution in a precipitation bath containing $75 \mathrm{wt} \%$ DMSO and $25 \mathrm{wt} \%$ water at various temperatures are shown in Fig. 2. It appears that 'delayed demixing' took place in all of the immersion cases. In particular, the light transmission intensity of the membrane solution at $65^{\circ} \mathrm{C}$ decreased by only $1 \%$ after immersion for $100 \mathrm{~s}$. This implies that the exchange rate between the coagulant and the solvent was very low in the precipitation medium.

\subsection{Membrane morphologies}

The SEM photomicrographs of a membrane prepared by immersing a $20 \mathrm{wt} \%$ EVAL solution in a precipitation bath containing $75 \mathrm{wt} \%$ DMSO and $25 \mathrm{wt} \%$ water at $25^{\circ} \mathrm{C}$ are shown in Fig. 3. This membrane exhibits a typical particulate morphology characteristic of EVAL crystallization $[9,15]$. Since the bath was very soft (75 wt\% DMSO), liquid-liquid demixing was sufficiently suppressed and crystallization dominated the precipitation process. From the light transmission experiment shown in Fig. 2, the composition change in the casting solution appeared to be slow. This implied that the DMSO arriving from the membrane interior to the surface has compensated the DMSO flowing into the bath. As a result, a flat concentration profile in the membrane was expected during the immersion-precipitation process in this soft bath [15]. And since the polymer volume fraction in the membrane bulk was essentially constant, nucleation of crystallization took place across the entire membrane solution virtually simultaneously. These nuclei then grew radially until their fronts met and joined with adjacent particles.

From the phase diagram of water-DMSO-EVAL at $25^{\circ} \mathrm{C}$ in Fig. 1, the binodal boundary is below the crystallization line, which provides, in terms of thermodynamics, a favorable condition for crystallization to occur earlier than liquid-liquid demixing. However, nucleation of liquid pores in liquid-liquid demixing is ordinarily more rapid than nucleation of polymer crystallites in crystallization since the latter requires rearrangements of the polymer chains into the crystalline lattice. Thus, the actual sequence of events in the phase separation process depends also on kinetic factors. It is possible that liquid-liquid demixing dominates the entire phase separation process, even though the binodal is inside the crystallization boundary. Such is the case when various dopes are immersed in the water bath $[9,15]$. As has been shown previously, the membrane thus formed exhibited a cellular structure derived from growth of liquid nuclei being surrounded by the polymer-rich phase. For the present immersion case, the bath contained a substantial amount of solvent so that before liquid-liquid phase separation could occur, crystallization commenced and the nascent membrane developed into the particulate structure. This is evident from the SEM pictures shown in Fig. 3, which comprises aggregated particles and the cellular pore is hardly observable. Therefore, it is sufficient for us to correlate, in the current delayed-demixing system, the membrane formation mechanism simply by thermodynamic factors without resorting to kinetics.

In Fig. 1, the crystallization line in the phase diagram 

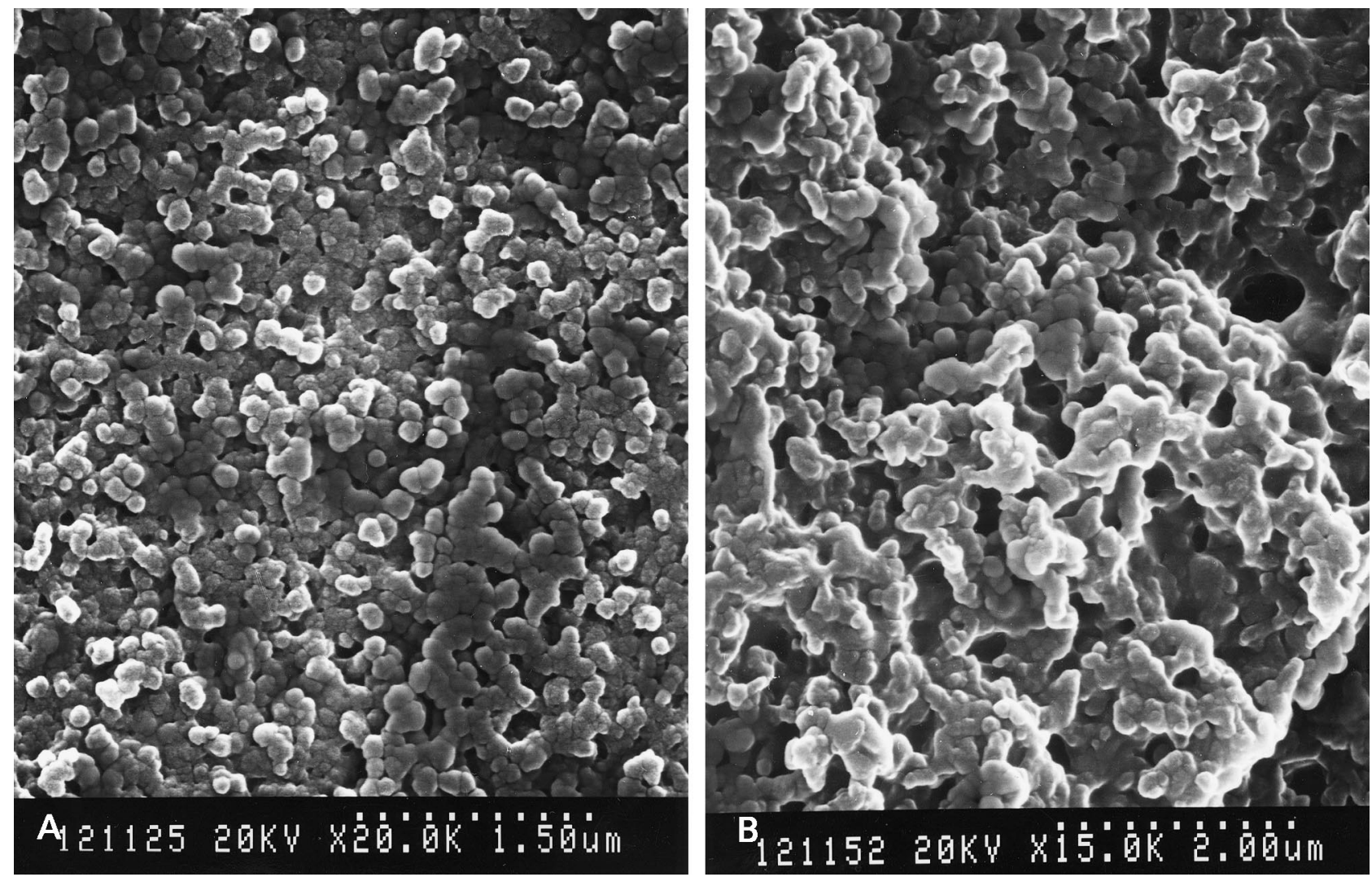

Fig. 3. SEM photomicrographs of a membrane prepared by immersing a $20 \mathrm{wt} \%$ EVAL solution in a precipitation bath containing $75 \mathrm{wt} \%$ DMSO and $25 \mathrm{wt} \%$ water at $25^{\circ} \mathrm{C}$. (A) top surface; (B) cross-section.

shifts toward the nonsolvent-polymer axis in a manner roughly parallel to that at $25^{\circ} \mathrm{C}$. Unlike the crystallization line which changes substantially with temperature, the binodal is affected by temperature only slightly. This leads to the fact that these two types of phase boundaries intersect each other at elevated temperatures. And there exists a region where liquid-liquid demixing can occur exclusively in the absence of crystallization. This implies that the casting membrane solution may enter into the binodal directly when the dope is immersed at $65^{\circ} \mathrm{C}$ in a precipitation bath containing $75 \mathrm{wt} \%$ DMSO and $25 \mathrm{wt} \%$ water. The measured tie line shown in Fig. 1 has the equilibrium composition of the dilute phase near the precipitating bath containing $75 \mathrm{wt} \%$ DMSO and that of the polymer-rich phase just below the intersection of crystallization line and binodal boundary, which provides an opportunity for a liquid-liquid demixing dominant precipitation process in this bath. This is evident in the SEM photomicrograph of the membrane shown in Fig. 4. The membrane has a skin layer on the top surface with a few small holes and demonstrates clearly the characteristics of a cellular morphology in the cross-section, while no particle structure resembling crystallization is observed. As the polymer solution was brought into contact with the precipitation bath by immersion, their interfaces soon reached equilibrium $[6,14,15]$. The composition of the membrane interface, being dictated by the equilibrium chemical potential, became close to the composition of the polymer-rich phase. This resulted in the possibility that the diffusion trajectory of the membrane solution might not enter the crystallization region, or the polymer-rich phase being slightly supersaturated with respect to crystallization, and that liquid-liquid demixing was the possible precipitation process in this bath condition.

The structure of the membrane prepared at $45^{\circ} \mathrm{C}$ using a $20 \mathrm{wt} \%$ EVAL solution in a precipitation bath containing $75 \mathrm{wt} \%$ DMSO and $25 \mathrm{wt} \%$ water at $45^{\circ} \mathrm{C}$ is shown in Fig. 5. This membrane also exhibits a cellular porous cross-section. However, the walls of the pores, unlike those observed in the membranes prepared at $65^{\circ} \mathrm{C}$, are composed of irregular particles. Such a structure represents the intermediate case between membranes prepared at 25 and $65^{\circ} \mathrm{C}$ (Figs 3 and 4, respectively). In other words, liquid-liquid demixing and crystallization were equally important during the membrane formation process. Since the pores are largely circular and regular, it was likely that liquid-liquid demixing had taken place first and before it was completed, polymer crystallization started at the pore wall $[11,15]$. It was also possible that liquid-liquid demixing and crystallization occurred almost simultaneously $[11,15]$. Such a result is consistent with the phase diagram shown in Fig. 1 . At $45^{\circ} \mathrm{C}$, the binodal boundary should be below the crystallization line but they are close to each other. This provides a condition for the binodal 

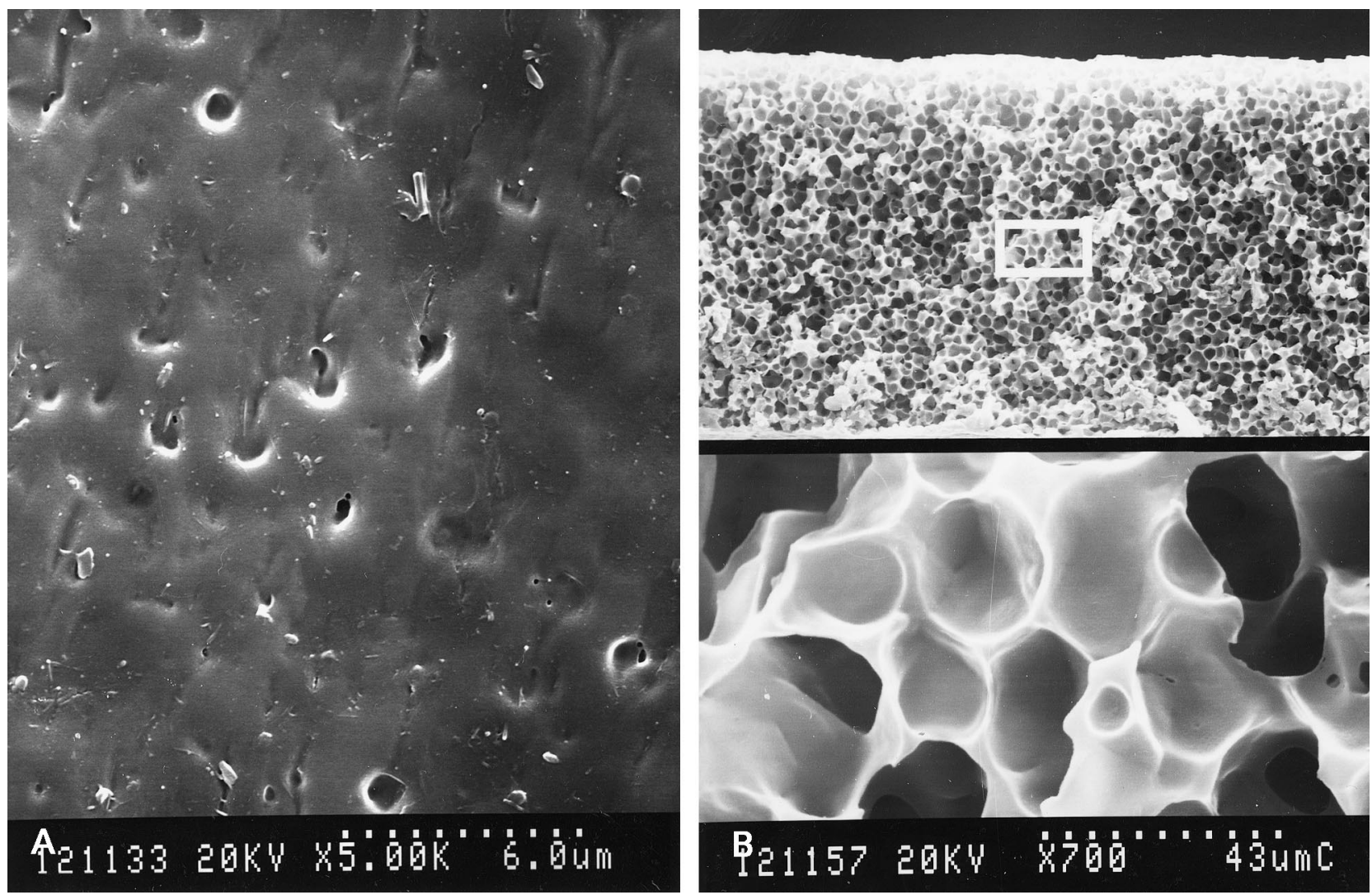

Fig. 4. The SEM photomicrographs of a membrane prepared by immersing a $20 \mathrm{wt} \%$ EVAL solution in a precipitation bath containing $75 \mathrm{wt} \%$ DMSO and $25 \mathrm{wt} \%$ water at $65^{\circ} \mathrm{C}$. (A) top surface; (B) cross-section.

to be entered and liquid-liquid demixing initiated before crystallization was initiated. As a result, the morphology formed exhibited features from both types of phase separations.

\section{Discussion}

The formation of a porous structure in a membrane is dictated by the sequence of the phase transition events in the precipitation process. When liquid-liquid demixing precedes crystallization, a cellular structure will be formed. On the other hand, crystallization may dominate the precipitation process to yield particulate morphology. Many parameters, such as the compositions of the casting solution and the precipitation bath and the temperature of the system, are known to exert particular influences on the morphology of the membranes formed. In this study, a variety of morphologies (ranging from cellular to particulate) were produced for the EVAL membranes by immersion-precipitation at different temperatures. In general, the structures of the precipitated membranes may be predicted by examining the thermodynamics (phase behavior) of the system and kinetics (mass transfer) in the immersion process. The phase diagram represents the ultimate equilibrium states in response to various interactions in the system. The mass transfer property mainly involves the rate of solvent and nonsolvent exchange relative to the polymer component. Therefore, the locations of crystallization and the liquid-liquid demixing boundaries, and the kinetics of these transitions will determine the weight of each transition on the final membrane morphology. Because crystallization is a slow process in comparison with liquid-liquid demixing, membranes form into a particulate morphology only in cases when liquid-liquid demixing is sufficiently suppressed, such as in systems with slow mass transfer rates.

The structures of EVAL membranes are more complicated than normal noncrystalline membranes because they contain characteristics derived from both the liquid-liquid demixing and crystallization processes. On the basis of the data presented in this report, it is obvious that by adjusting the immersion temperature, the membrane structure can be effectively modified in coagulation baths having a high solvent content. In such a bath, the exchange rate between coagulant and solvent is slow; hence, before the membrane solution enters the binodal to initiate liquid-liquid demixing, there exists a period of time long enough for the nucleation of crystallization to be inducted. This leads to the formation of a large variety of morphologies with different contents of both types of phase separation. At $25^{\circ} \mathrm{C}$, the membranes formed exhibit a particulate structure (characteristic of crystallization), whereas at $65^{\circ} \mathrm{C}$ a cellular structure (characteristic of liquid-liquid demixing) is 

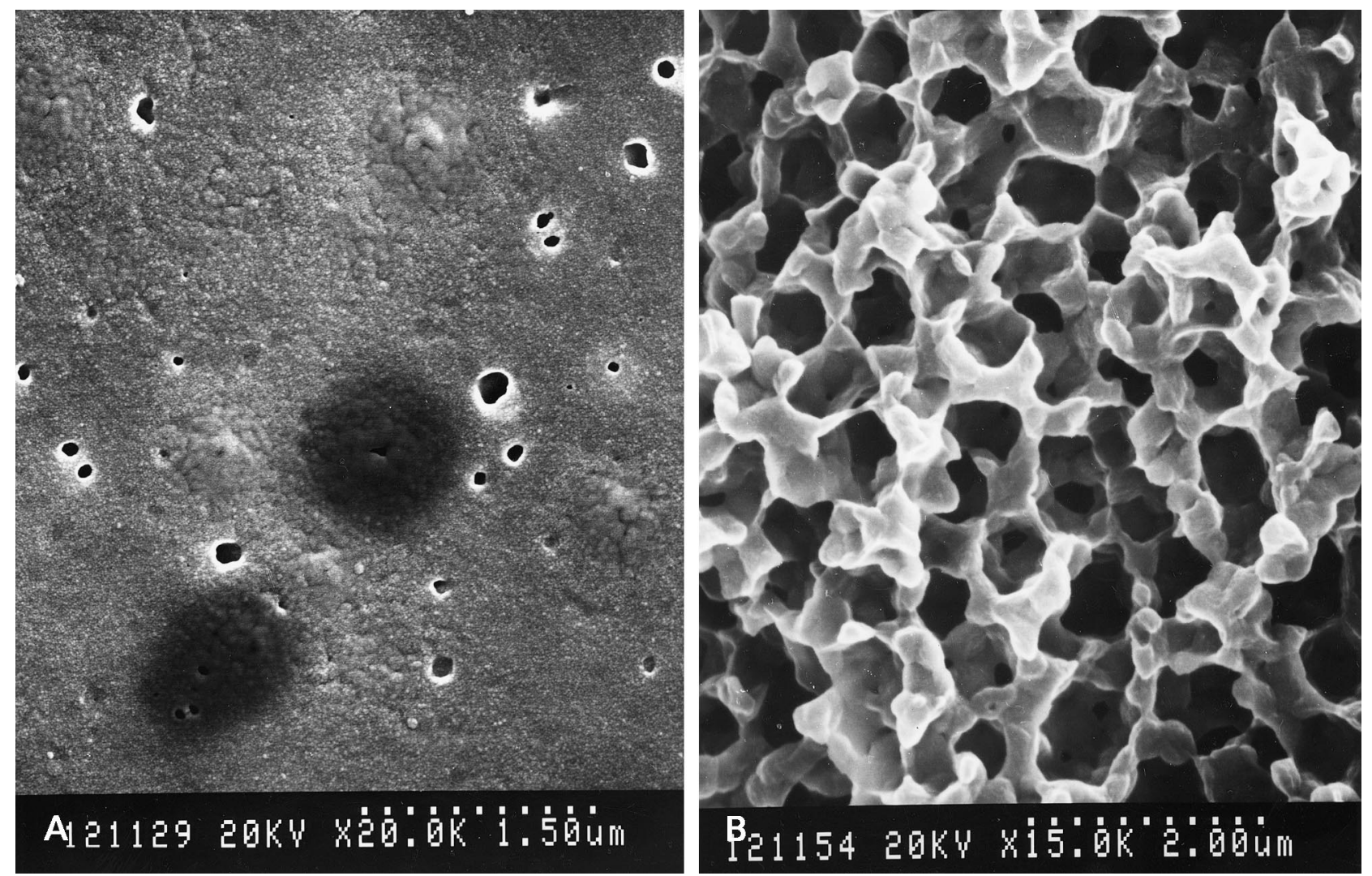

Fig. 5. The SEM photomicrographs of a membrane prepared by immersing a $20 \mathrm{wt} \%$ EVAL solution in a precipitation bath containing $75 \mathrm{wt} \%$ DMSO and $25 \mathrm{wt} \%$ water at $45^{\circ} \mathrm{C}$. (A) top surface; (B) cross-section.

obtained. Such a system is thermodynamically controlled and a good correlation between the phase diagram and membrane morphology is established. In contrast, only liquid-liquid demixing may occur during membrane formation for amorphous polymers. Therefore, since the binodals are less sensitive to temperature changes in the phase diagram, the structures of noncrystalline membranes will not have significant changes at different temperatures, such as EVAL membranes having a temperature switch between particulate and cellular morphologies.

\section{Acknowledgements}

The authors thank the National Science Council of Taiwan, the Republic of China for their financial support of this research, NSC 85-2216-E-032-001 and NSC 85-2216-E-002-002.

\section{References}

[1] Kesting, RE. Synthetic polymeric membranes. New York: Wiley, 1985.

[2] Mulder, M. Basic principles of membrane technology Dordrecht: Kluwer, 1991.

[3] Lloyd DR, Kinzer KE, Tseng HS. Microporous membrane formation via thermally induced phase separation. I. Solid-liquid phase separation. J Membrane Sci 1992;52:239-261.

[4] Bulte AMW, Folkers B, Mulder MHV, Smolders CA. Membranes of semicrystalline aliphatic polyamide nylon 4,6: formation by diffusioninduced phase separation. J Appl Polym Sci 1993;50:13.

[5] Cheng LP, Dwan AW, Gryte CC. Membrane formation by isothermal precipitation in polyamide-formic acid-water systems I. Description of membrane morphology. J Polym Sci, Polym Phys 1995;33:211.

[6] Cheng LP, Dwan AW, Gryte CC. Membrane formation by isothermal precipitation in polyamide-formic acid-water systems II. Precipitation dynamics. J Polym Sci, Polym Phys 1995;33:223.

[7] Bulte AMW, Mulder MHV, Smolders CA, Strathmann H. Diffusion induced phase separation with crystallizable nylons. I. Mass transfer processes for nylon 4,6. J Membrane Sci 1996;121:37-49.

[8] van de Witte P, Esselbrugge H, Dijkstra PJ, van de Berg JWA, Feijen J. A morphological study of membranes obtained from the systems polylactidedioxane-methanol, polylactide-dioxane-water, and polylactide-N-methyl pyrrolidone-water. J Polym Sci Polym Phys 1996;34:2569-2578.

[9] Young TH, Lai JY, You WM, Cheng LP. Equilibrium phase behavior of the membrane forming water-DMSO-EVAL copolymer system. J Membrane Sci 1997;128:55-65.

[10] Young TH, Cheng LP, Hsieh CC, Chen LW. Phase behavior of EVAL polymer in water-2-propanol cosolvent. Macromolecules, 1998;31 1221-1235.

[11] Young TH, Wang DM, Hsieh CC, Chen LW. The effect of the second phase inversion on microstructures in phase inversion EVAL membranes. J Membrane Sci, in press.

[12] Cheng LP, Lin HI, Chen LW, Young TH. Solute rejection of EVAL membranes with asymmetric and microparticulate morphologies. Polymer 1998;39:2135-2142. 
[13] Lin DT, Cheng LP, Kang YJ, Chen LW, Young TH. Effects of precipitation conditions on the membrane morphology and permeation characteristics. J Membrane Sci, 1998;140:185-194.

[14] Reuvers AJ, van der Berg JWA, Smolders CA. Formation of membranes by means of immersion precipitation. Part I. A model to describe mass transfer during immersion precipitation. J Membrane Sci 1987;34:45-65.

[15] Cheng LP, Young TH, You YM. Formation of crystalline EVAL membranes by controlled mass transfer process in water-DMSOEVAL copolymer systems. J Membrane Sci 1998;145:77-90. 